\title{
(a) OPEN ACCESS \\ Microaneurysms visualisation using five different optical coherence tomography angiography devices compared to fluorescein angiography
}

\author{
Salvatore Parrulli, Federico Corvi (1D, Mariano Cozzi, Davide Monteduro, \\ Federico Zicarelli, Giovanni Staurenghi
}

Eye Clinic, Department of Biomedical and Clinical Sciences Luigi Sacco, University of Milan, Milano, Italy

\section{Correspondence to} Dr Federico Corvi, Eye Clinic, Department of Biomedical and Clinical Science 'Luigi Sacco', Sacco Hospital, University of Milan, Via G.B Grassi 74, Milan, 20157, Italy;

federico.corvi@yahoo.it

SP and FC contributed equally.

Received 4 May 2020 Revised 25 May 2020

Published Online First 10 June 2020

\section{Check for updates}

(C) Author(s) (or their employer(s)) 2021. Re-use permitted under CC BY-NC. No commercial re-use. See rights and permissions. Published by BMJ.

To cite: Parrulli S, Corvi F,

Cozzi M, et al.

Br J Ophthalmol

2021:105:526-530.

\section{ABSTRACT}

Background To compare fluorescein angiography (FA) and five different optical coherence tomography angiography (OCTA) devices and to test their reproducibility in the evaluation of retinal microaneurysms (MAs) secondary to diabetic retinopathy (DR).

Methods On the same day, patients with DR were imaged with FA and five OCTA devices: prototype Spectralis OCTA, prototype PlexElite, RTVue XR Avanti, AngioPlex and DRI OCT Triton. For all OCTA devices, a $3 \times 3$ volume scan pattern was performed. MAs were evaluated for the superficial capillary plexus (SCP) and deep capillary plexus (DCP).

Results Twenty eyes of 15 patients with DR were included. FA counted a significantly higher number of MAs compared to OCTA devices. Spectralis OCTA obtained a significantly higher number of MAs compared to PlexElite, RTVue XR Avanti, AngioPlex and DRI OCT Triton $(p<0.0001)$. PlexElite and AngioPlex showed a greater number of MAs in the SCP, Spectralis OCTA, RTVue XR Avanti and DRI OCT Triton in the DCP. Higher sensitivity (43.3\%) but lowest specificity (54.4\%) was observed for Spectralis OCTA compared to other devices. The higher specificity $(78.5 \%)$ and positive predictive value $(83.3 \%)$ were observed for DRI OCT Triton.

Conclusions FA remains the best imaging modality to visualise retinal MAs. Spectralis OCTA was able to detect more MAs compared to other devices, likely due to the higher number of B-scans in the scanned area as well as due to the higher number of repeated B-scans. The high variability between OCTA devices should be taken into account for future clinical trials as in clinical practice.

\section{INTRODUCTION}

Diabetic retinopathy (DR) is the major cause of vision impairment and blindness among individuals in working age. ${ }^{1}$ By 2030 , the number of patients with diabetes worldwide is estimated to be 429 million, and it will continue to grow due to lifestyle, obesity and higher disease detection rate. ${ }^{2}$

Generally, microaneurysms (MAs) are the early pathologic signs in patients with DR. ${ }^{2}$ Several histopathologic studies described MAs as dilations of capillaries, more numerous in the central retina and the great part of them originating from the deep capillary plexus (DCP) and in the inner nuclear layer. $^{3-5}$ In particular, their formation is a consequence of the thickening of basement membrane, loss of pericytes and capillary endothelial cell dysfunction. ${ }^{1}$ The formation of diabetic macular oedema is related to the leakage from MAs and the breakdown of blood-retinal barrier with high concentrations of cytokines. ${ }^{67}$ In this contest, MAs are an essential sign for diagnosis and staging of DR.

Historically, colour fundus photography has been widely used in clinical trials and daily practice. ${ }^{8-10}$ However, MAs appear as red dots and could be not distinguished by little dot haemorrhages. For this reason, fluorescein angiography (FA) has been considered the gold standard for detection of MAs, visualised as hyperfluorescent dots on early phases. $^{11}$

A recent imaging modality is optical coherence tomography angiography (OCTA) that offers the opportunity to visualise different retinal capillary plexus and the choroidal vasculature without the need for dye injection. ${ }^{5} 12$ Although it has been mainly used in the detection of choroidal neovascularisation, many other retinal diseases have been investigated. ${ }^{5}{ }^{13}$ In DR, this imaging modality was used to evaluate the non-perfusion areas, choroidal alterations, retinal neovascularisation and MAs. ${ }^{14}$

It should be considered that OCTA is currently used into clinical trials of patients with DR. However, there are several commercial OCTA using different algorithms, wavelengths and scan patterns. For this reason, studies are needed to evaluate whether quantifiable metrics applied to the vasculature on OCTA are comparable between devices. In this context, several studies investigated the reproducibility of several OCTA on vessel density, fractal dimension and foveal avascular zone in healthy volunteers as well as quantitative parameters in choroidal neovascularisations. ${ }^{15} 16$ The purpose of this study is to compare FA and five different OCTA devices and to test their reproducibility in the evaluation of MAs.

\section{METHODS}

This is an inter-reliability analysis in patients with DR presenting at the Eye Clinic, Department of Biomedical and Clinical Sciences, Luigi Sacco Hospital, University of Milan, from 1 April 2019 to 31 May 2019. The study was approved by the Luigi Sacco Hospital Ethics Committee in Milan and adhered to the tenets of the Declaration of Helsinki for research involving human subjects.

Consecutive patients with DR were enrolled and an informed consent was obtained by all subjects. Inclusion criteria were (1) age greater than 18 years and (2) presence of DR diagnosed by fundus 
examination. Exclusion criteria were (1) the presence of MAs secondary to diseases other than DR, (2) choroidal neovascularisation, (3) central serous chorioretinopathy, (4) retinal vein occlusion, (5) diffuse macular oedema (defined as a retinal thickness $>350 \mu \mathrm{m})$ and (6) presence of significant media opacities that could reduce image quality.

All patients underwent a complete ophthalmologic evaluation including best-corrected visual acuity, tonometry, slit lamp and fundus examination after pupil dilation. The following imaging procedures were performed on the same day in all patients: colour fundus photography DRI OCT Triton (Topcon, Tokyo, Japan), spectral-domain optical coherence tomography (Spectralis October-2, Heidelberg Engineering, Heidelberg, Germany), FA (Spectralis HRA 2, Heidelberg Engineering) and five different OCTA devices: prototype Spectralis OCTA (Version SP 6.9a; Spectralis, Heidelberg Engineering), prototype PlexElite (Version 1.7.1.31492; Carl Zeiss Meditec, Dublin, California, USA), RTVue XR Avanti (2017.1.0.150; Optovue, Fremont, California, USA), AngioPlex (Version 11.0.0.29946; Cirrus 5000 HD-OCT, Carl Zeiss Meditec) and DRI OCT Triton (IMAGEnet6 version 1.24.1; Topcon) (table 1).

\section{Imaging analysis}

In all cases, a $3 \times 3 \mathrm{~mm}$ volume pattern centred on the fovea was acquired by an experienced operator (MC). To be included, the scans had to have sufficient image quality as AngioPlex and PlexElite with a signal strength index $>7$ (range 0-10), Spectralis OCTA with a signal-to-noise ratio $>25 \mathrm{~dB}$ (range $0-40$ ), RTVue XR Avanti with a quality score $>70$ (range $0-100$ ) and DRI OCT Triton with a quality index $>70$ (range $0-100)$ and no motion artefact.

The default automated segmentation boundaries were used in order to obtain the images of the superficial capillary plexus (SCP) and DCP. All projection artefacts were removed using the default function for each device. A squared region of interest centred on the fovea was used in order to avoid any influence by the non-proper overlapping between images.
The evaluation of MAs on OCTA images was performed by two blinded readers (FC and SP), who recognised MAs as saccular, fusiform or focal bulge originating from a retinal vessel, as previously described. ${ }^{4}$ Disagreements between readers were arbitrated by an independent senior reader (GS). FA was considered the gold standard to assess the presence of MAs. For this reason, OCTA images were overlapped to FA images in the early phases (within $1 \mathrm{~min}$ from dye injection) in order to detect MAs. Late FA images (at least 10 min after dye injection) were necessary to define the leakage from MAs. Each MA identified in the SCP and DCP of different OCTA devices was graded and named in order to recognise which of them was nonvisible or localised in the superficial layer, deep layer or both (figure 1).

\section{Statistical analysis}

Statistical calculations were performed using Statistical Package for the Social Sciences (v.22.0, IBM Corp, Armonk, New York, USA). Data were expressed as mean \pm SD. Sensitivity, specificity, positive predictive value (PPV) and negative predictive value (NPN) were calculated for each OCTA device. Sensitivity was computed as number of true positives/number of true positives + number of false negatives; specificity as number of true negatives/number of true negatives + number of false positives; PPV as number of true positives/number of true positives + number of false positives; NPV as number of true negatives/number of true negatives + number of false negatives. The comparison between different devices was performed evaluating the presence or the absence of each corresponding MA using Cochran's Q test followed by post hoc analysis using McNemar correction for multiple comparisons and evaluating the total number of MAs using Wilcoxon test. Binary logistic regression analysis was performed to evaluate the influence of leakage on the visibility of MAs on OCTA. The chosen level of statistical significance was $p<0.05$. Cohen's kappa coefficient was calculated to quantify the intergrader agreement for ordered categories.

Table 1 Optical coherence tomography angiography devices

\begin{tabular}{|c|c|c|c|c|c|c|c|c|}
\hline Device & Software version & Wavelength $(\mathrm{nm})$ & Algorithm & A-scan/B-scan & Vascular plexus & Slab boundary & Anatomic basis & Offset \\
\hline $\begin{array}{l}\text { Spectralis } \\
\text { OCT-A } \\
\text { (Heidelberg } \\
\text { Engineering) }\end{array}$ & SP 6.12 & 870 & $\begin{array}{l}\text { Probabilistic } \\
\text { OCT-A algorithm }\end{array}$ & $512 / 512$ & $\begin{array}{l}\text { SCP } \\
\text { DCP }\end{array}$ & $\begin{array}{l}\text { Top } \\
\text { Bottom } \\
\text { Top } \\
\text { Bottom }\end{array}$ & $\begin{array}{l}\text { ILM } \\
\text { IPL } \\
\text { IPL } \\
\text { OPL }\end{array}$ & $\begin{array}{l}0 \\
0 \\
0 \\
0\end{array}$ \\
\hline $\begin{array}{l}\text { PlexElite } \\
\text { (Carl Zeiss } \\
\text { Meditec) }\end{array}$ & 1.7.1.31492 & 1060 & OMAG & $300 / 300$ & $\begin{array}{l}\text { SCP } \\
\text { DCP }\end{array}$ & $\begin{array}{l}\text { Top } \\
\text { Bottom } \\
\text { Top } \\
\text { Bottom }\end{array}$ & $\begin{array}{l}\text { ILM } \\
\text { IPL } \\
\text { IPL } \\
\text { OPL }\end{array}$ & $\begin{array}{l}0 \\
0 \\
0 \\
0\end{array}$ \\
\hline $\begin{array}{l}\text { Angioplex } \\
\text { (Carl Zeiss } \\
\text { Meditec) }\end{array}$ & 11.0 .0 .29946 & 840 & OMAG & $245 / 245$ & $\begin{array}{l}\text { SCP } \\
\text { DCP }\end{array}$ & $\begin{array}{l}\text { Top } \\
\text { Bottom } \\
\text { Top } \\
\text { Bottom }\end{array}$ & $\begin{array}{l}\text { ILM } \\
\text { IPL } \\
\text { IPL } \\
\text { OPL }\end{array}$ & $\begin{array}{l}0 \\
0 \\
0 \\
0\end{array}$ \\
\hline
\end{tabular}

DCP, deep capillary plexus; ILM, internal limiting membrane; IPL, inner plexiform layer; OCTA, optical coherence tomography angiography; OCTARA, OCTA ratio analysis; OMAG, optical microangiography; OPL, outer plexiform layer; SCP, superficial capillary plexus; SSADA, Split-Spectrum Amplitude-Decorrelation Algorithm. 

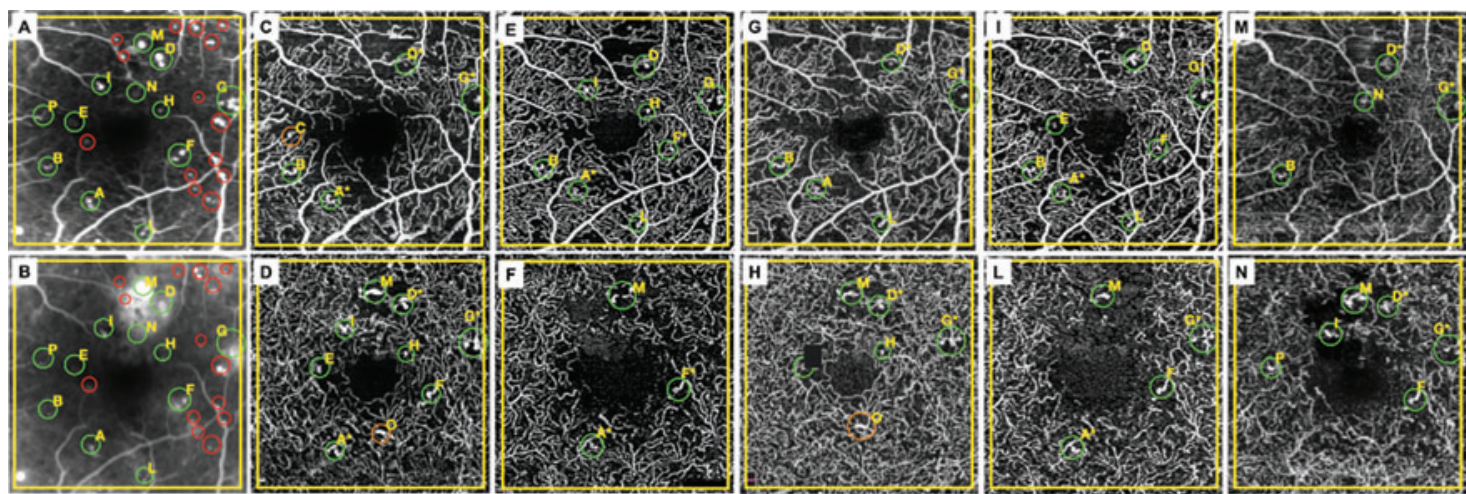

Figure 1 From left to right, each column corresponds to early $(A)$ and late $(B)$ phase of fluorescein angiography, superficial (C, E, G, I, M) and deep ( $D, F$, $\mathrm{H}, \mathrm{L}, \mathrm{N}$ ) capillary plexus of Spectralis OCTA, PlexElite, RTVue XR Avanti, AngioPlex and DRI OCT Triton, respectively. An univocal letter is assigned to each microaneurysm (MA) detected by fluorescein angiography and optical coherence tomography angiography. Green circles indicate each MA detected on OCTA and FA. Orange circles indicate each MA detected on OCTA and not on FA. Red circles represent each MAs detected on FA and not on OCTA. FA, fluorescein angiography; OCTA, optical coherence tomography angiography.

\section{RESULTS}

A total of 30 eyes from 15 patients with DR were included in this study. Among these 30 eyes, 10 eyes were excluded due to poor image quality or significant artefacts in at least one OCTA device, and thus ultimately 20 eyes were included in the analysis. The demographic features are listed in table 2. The number of MAs counted in the SCP and DCP for each instrument and for FA is presented in table 3 . FA counted the highest number of MAs with a significant difference compared to all the OCTA devices

\begin{tabular}{|c|c|}
\hline Demographic features & $\mathrm{N}(\%)$ \\
\hline \multicolumn{2}{|l|}{ Age (years) ( $n=13$ patients) } \\
\hline Mean \pm SD (range) & $67.8 \pm 8.1(58-80)$ \\
\hline \multicolumn{2}{|l|}{ Race ( $n=13$ patients) } \\
\hline Caucasian & $11(84)$ \\
\hline Asian & $1(8)$ \\
\hline Hispanic & $1(8)$ \\
\hline African-American & $0(0)$ \\
\hline \multicolumn{2}{|l|}{ Gender ( $n=13$ patients) } \\
\hline Men & $11(84)$ \\
\hline Women & $2(16)$ \\
\hline \multicolumn{2}{|c|}{ Duration of diabetes (years) ( $n=13$ patients) } \\
\hline Mean \pm SD (range) & $9.57 \pm 4.2(4-17)$ \\
\hline \multicolumn{2}{|l|}{ Eye involved ( $n=20$ eyes) } \\
\hline Unilateral & $7(54)$ \\
\hline Bilateral & $6(46)$ \\
\hline \multicolumn{2}{|l|}{$\mathrm{HbA1c}(\%)(\mathrm{n}=13$ patients $)$} \\
\hline Mean \pm SD (range) & $7.8 \pm 0.84(6.8-9.1)$ \\
\hline \multicolumn{2}{|c|}{ International Clinical DR Disease Severity Scale ( $\mathrm{n}=20$ eyes) } \\
\hline NPDR mild & $7(35)$ \\
\hline NPDR moderate & $4(20)$ \\
\hline NPDR severe & $1(5)$ \\
\hline PDR active & $0(0)$ \\
\hline PDR inactive & $8(40)$ \\
\hline \multicolumn{2}{|l|}{ BCVA (LogMar) } \\
\hline Mean $\pm S D$ (range) & $0.31 \pm 0.20(0.70-0.10)$ \\
\hline
\end{tabular}

$(\mathrm{p}<0.0001)$. Binary logistic regression analysis did not show any association between leakage and the visibility of MAs on OCTA.

Considering both SCP and DCP, Spectralis OCTA obtained a significantly higher number of MAs compared to PlexElite, RTVue XR Avanti, AngioPlex and DRI OCT Triton $(p<0.0001)$. No significant differences were found between PlexElite, RTVue XR Avanti, AngioPlex and DRI OCT Triton $(p>0.05)$. In the SCP, significant differences were found for Spectralis OCTA compared to RTVue XR Avanti and DRI OCT Triton ( $p=0.002$ and $p=0.001$, respectively), for PlexElite compared to RTVue XR Avanti and DRI OCT Triton $(\mathrm{p}<0.0001$ and $\mathrm{p}=0.001$, respectively) and between AngioPlex and DRI OCT Triton $(\mathrm{p}=0.013)$. All the other comparisons between the OCTA

Table 3 Microaneurysms visualised by different optical coherence tomography angiography devices and fluorescein angiography

\begin{tabular}{llll}
\hline Device & $\begin{array}{l}\text { Vascular } \\
\text { plexus }\end{array}$ & $\begin{array}{l}\text { Total number of } \\
\text { MAs }\end{array}$ & $\begin{array}{l}\text { Mean number of } \\
\text { MAs }\end{array}$ \\
\hline Spectralis OCTA & SCP & $124(39 \%)$ & $6.2 \pm 3.9$ \\
& DCP & $198(61 \%)$ & $9.9 \pm 3.7$ \\
& SCP + DCP & 322 & $16.1 \pm 6.4$ \\
\# SCP + DCP & 293 & $/$ \\
PlexElite & SCP & $133(65 \%)$ & $6.6 \pm 2.7$ \\
& DCP & $72(35 \%)$ & $3.6 \pm 2.3$ \\
& SCP + DCP & 205 & $10.2 \pm 4.5$ \\
RTVue XR & \# SCP + DCP & 190 & $/$ \\
& SCP & $83(44 \%)$ & $4.1 \pm 2.2$ \\
& DCP & $104(56 \%)$ & $5.2 \pm 2.3$ \\
& SCP + DCP & 187 & $9.3 \pm 3.4$ \\
AngioPlex & \# SCP + DCP & 170 & $/$ \\
& SCP & $113(70 \%)$ & $5.6 \pm 2.5$ \\
& DCP & $48(30 \%)$ & $2.4 \pm 1.6$ \\
& SCP + DCP & 161 & $8.1 \pm 3.8$ \\
DRI OCT Triton & \# SCP + DCP & 149 & $/$ \\
& SCP & $76(43 \%)$ & $3.8 \pm 2.1$ \\
& DCP & $102(57 \%)$ & $5.1 \pm 2.8$ \\
& SCP + DCP & 178 & $8.9 \pm 3.5$ \\
Fluorescein & \# SCP + DCP & 161 & $/$ \\
angiography & SCP + DCP & 550 & $/$ \\
\hline DCP, dep cap & & & \\
\hline
\end{tabular}

DCP, deep capillary plexus; MAs, microaneurysms; OCTA, optical coherence tomography angiography; $S C P$, superficial capillary plexus: $S C P+D C P$, sum of total microaneurysms for SCP and DCP; \# SCP + DCP; sum of microaneurysms visualised in the SCP and DCP considering as a single unit which is visualised in both plexuses. 
devices were not significantly different $(p>0.05)$. In the DCP, the comparisons between the OCTA devices were significantly different $(\mathrm{p}<0.05)$ except between RTVue XR Avanti and DRI OCT Triton $(\mathrm{p}=0.858)$.

Moreover, we evaluated MAs as single units and compared each MA between FA and the different OCTA devices considering the SCP and DCP. In SCP and DCP, significant differences were found for Spectralis OCTA compared to the other studied OCTA devices $(p<0.0001)$ and for PlexElite compared to AngioPlex and DRI OCT Triton ( $\mathrm{p}=0.02$ and $\mathrm{p}=0.031$, respectively). In the SCP, significant differences were found for DRI OCT Triton compared to Spectralis OCTA, PlexElite and AngioPlex $(\mathrm{p}<0.0001, \mathrm{p}<0.0001$ and $\mathrm{p}=0.001$, respectively $)$ and for RTVue XR Avanti compared to Spectralis OCTA, PlexElite and AngioPlex ( $<<0.0001, p<0.0001$ and $p=0.013$, respectively). In the DCP, the comparisons between the OCTA devices were significantly different $(\mathrm{p}<0.05)$ except between RTVue XR Avanti with DRI OCT Triton $(\mathrm{p}=0.863)$. Furthermore, we computed the sensitivity, specificity, PPV and NPV with their 95\% CIs for each OCTA device as shown in table 4.

At least, for the intergrader reliability analysis, a Cohen's kappa was calculated for each instrument (FA k=0.98, Spectralis OCTA $\mathrm{k}=0.95$, PlexElite $\mathrm{k}=0.93$, RTVue XR Avanti $\mathrm{k}=0.89$, AngioPlex k=0.90, DRI OCT Triton $\mathrm{k}=0.88$ ).

\section{DISCUSSION}

OCTA has a central role in the daily practice of retina specialists, and growing importance is now given in clinical trials, thanks to its non-invasiveness and the high-quality visualisation of retinal and choroidal vessels. ${ }^{12}{ }^{17}$ Interest is growing about the way OCTA could help visualising vascular abnormalities in DR. In this study, we investigated the ability of five different OCTA devices to detect MAs and compare these findings to FA.

FA obtained the best results in order to visualise MAs and offered the opportunity to detect the eventual associated leakage of the dye. In particular, 550 MAs were counted by FA, a number significantly higher than all OCTA devices. These results were similar to those observed in previous studies comparing these imaging modalities. ${ }^{18} 19$ It is well known that the blood flow detectable by OCTA depends on the flow speed, the intramicroaneurysmal turbulence and the interscan time, also known as the time between two B-scans. ${ }^{520}$ If the blood flow is too low, it will not produce a decorrelation signal. At the same time, the ability to detect blood flow is strictly related to the interscan time. Longer interscan time may allow the detection of very slow flow, while a shorter interscan time is associated with less sensitivity in

Table 4 Reliability analysis

\begin{tabular}{lllll}
\hline & $\begin{array}{l}\text { Sensibility } \\
(95 \% \mathrm{Cl})\end{array}$ & $\begin{array}{l}\text { Specificity } \\
(\mathbf{9 5 \%} \mathrm{Cl})\end{array}$ & $\begin{array}{l}\text { Positive predic- } \\
\text { tive value } \\
(\mathbf{9 5 \%} \mathrm{Cl})\end{array}$ & $\begin{array}{l}\text { Negative predic- } \\
\text { tive value } \\
(95 \% \mathrm{CI})\end{array}$ \\
\hline Spectralis & $43.3 \%$ & $54.5 \%$ & $81.2 \%$ & $17.4 \%$ \\
OCTA & $(39.1-47.5 \%)$ & $(45.2-63.6 \%)$ & $(77.7-84.3 \%)$ & $(15.1-20.1 \%)$ \\
\hline PlexElite & $27.8 \%$ & $69.4 \%$ & $80.5 \%$ & $17.4 \%$ \\
& $(24.1-31.8 \%)$ & $(60.4-77.5 \%)$ & $(75.4-84.8 \%)$ & $(15.7-19.4 \%)$ \\
RTVue XR & $24.2 \%$ & $69.4 \%$ & $78.2 \%$ & $16.7 \%$ \\
& $(20.7-28 \%)$ & $(60.4-77.5 \%)$ & $(72.6-83 \%)$ & $(15.1-18.6 \%)$ \\
AngioPlex & $20.9 \%$ & $71.9 \%$ & $77.1 \%$ & $16.1 \%$ \\
& $(17.6-24.5 \%)$ & $(63-79.7 \%)$ & $(70.9-82.4 \%)$ & $(15.1-18.4 \%)$ \\
DRI OCT & $24.5 \%$ & $78.5 \%$ & $83.8 \%$ & $18.6 \%$ \\
Triton & $(21.0-28.3 \%)$ & $(70.1-85.4 \%)$ & $(78.2-88.3 \%)$ & $(17.1-20.3 \%)$ \\
\hline
\end{tabular}

OCTA, optical coherence tomography angiography. detecting slow flow. The possible explanation for the lower number of MAs visualised by OCTA may be related to the blood flow that could be slow or even turbulent, with a consequent missing decorrelation signal. Turbulent MAs may not be detected by OCTA due to the change in plasma flow or erythrocytes. It means even using the same device on the same subject, detection of MAs can be variable. ${ }^{20}$ Furthermore, we evaluated the impact of leakage in the visualisation of MAs by OCTA and we found that leakage of MAs is not associated with a higher visualisation rate on OCTA images, independently of the device used. It is likely that the blood flow inside the vascular walls is the only one responsible for a decorrelation signal. This result is in contrast to Schreur $e t$ al $^{18}$ that reported an improved visualisation of MAs by OCTA in the areas of focal leakage and macular oedema. However, in our study, we did not include patients with frank macular oedema as we wanted to minimise the bias due to the incorrect automatic segmentation.

Considering OCTA devices in the evaluation of the total number of MAs, we found significant differences between Spectralis OCTA and the other devices in both the SCP and DCP, and no differences were found between the other devices. Similarly, considering MAs as single units, we found significant differences between Spectralis OCTA and the other devices, between PlexElite and AngioPlex, between PlexElite and DRI OCT Triton in both the SCP and DCP. These results could be explained by the different segmentation used by Spectalis comparing with the others where the first is using a flow signal to differentiate the retinal vascular layers and the others the structural OCT layers.

In this context, we have to consider the distribution of MAs in the capillary plexus. Spectralis OCTA, RTVue XR and DRI OCT Triton counted more than $55 \%$ of MAs in the DCP, while PlexElite and AngioPlex detected more than 65\% of MAs in the SCP. Couturier et $a l^{19}$ evaluated the visualisation of MAs using RTVue XR Avanti and FA, and found that fewer MAs were visualised by OCTA compared to FA and more MAs were located in the DCP. Hasegawa et $a l^{21}$ using RTVue XR Avanti found that $77.3 \%$ of all MAs were located in the DCP in eyes without macular oedema and $91.3 \%$ in eyes with macular oedema $>400 \mu \mathrm{m}$.

In our study, we found that PlexElite and AngioPlex showed a higher number of MAs in the SCP and Spectralis OCTA, RTVue XR Avanti and DRI OCT Triton in the DCP. One of the possible explanations of these findings is the position of the segmentation boundaries for the SCP and DCP between the OCTA devices. However, we found differences between devices using the same segmentation boundaries and also the same decorrelation algorithm. Two other possible factors that should be taken into account are the different number of B-scans and the different number of repeated B-scans in the acquisition protocol by OCTA devices that may change the visualisation of retinal capillaries and, consequently, of MAs. ${ }^{5} 16$ Lastly, we could not exclude the role of the algorithm used to originate the flow signal as well as the flow signal threshold of each device. The combination of all these aspects, including different wavelengths, acquisition patterns, decorrelation algorithms and postprocessing elaboration, may explain the differences in the visualisation of MAs.

All OCTA devices showed a similar PPV. This should be interpreted as a possibility close to $80 \%$ that an MA detected on OCTA may correspond to a true MA detected on FA. In this context, considering the analysis of sensitivity and specificity, we found that Spectralis OCTA obtained greater sensitivity compared to other instruments although it is associated with a lower 
specificity. On the other side, DRI OCT Triton obtained greater specificity and PPV. These results should be interpreted as the ability of Spectralis OCTA in detecting a higher number of MAs, although some of these are false positive, with a subsequent reduction of specificity. To our knowledge, this is the first time a complete reliability analysis is performed about OCTA ability to visualise MAs. Several other previous studies were restricted to show in percentage the amount of MAs on FA that were recognisable on OCTA, with high variations among different analyses, varying from $34 \%$ to $84 \% .{ }^{19} 22$

This study presents several limitations. This study included a small sample of patients, although associated with a high number of MAs detected. Moreover, we did not perform multiple acquisitions by the same device that may increase the image quality and may improve the visibility of MAs. ${ }^{22}$ However, a high level of patient compliance and long acquisition times are essential to perform multiple scans with the same device and with multiple devices.

In conclusion, we compared five different OCTA devices and traditional FA in the ability to detect retinal MAs. FA appeared to be the best imaging modality to identify retinal MAs. Spectralis OCTA was able to detect more MAs compared to other devices, likely due to the higher number of B-scans in the scanned area as well as due to the higher number of repeated B-scans. Different OCTA devices showed a higher number of MAs in the SCP while others in the DCP. The high variability between OCTA devices should be taken into account for future clinical trials as in clinical practice.

Contributors SP and FC have contributed to study conception, design, analysis, data acquisition, interpretation of data, draf and revision, and final approval. MC, DM and FZ have contributed to data acquisition, revision and final approval. GS have contributed to interpretation of data, draft and revision, and final approval.

Funding This research received no specific grant from any funding agency in the public, commercial or not-for-profit sectors.

Competing interests None declared.

Data sharing statement All data relevant to the study are included in the article or uploaded as supplementary information.

Provenance and peer review Not commissioned; externally peer reviewed.

Open access This is an open access article distributed in accordance with the Creative Commons Attribution Non Commercial (CC BY-NC 4.0) license, which permits others to distribute, remix, adapt, build upon this work non-commercially, and license their derivative works on different terms, provided the original work is properly cited, appropriate credit is given, any changes made indicated, and the use is noncommercial. See: http://creativecommons.org/ licenses/by-nc/4.0/.

ORCID ID

Federico Corvi http://orcid.org/0000-0002-2661-5500

\section{REFERENCES}

1 Durham JT, Herman IM. Microvascular modifications in diabetic retinopathy. Curr Diab Rep 2011;11:253-64.

2 Antonetti DA, Klein R, Gardner TW. Diabetic retinopathy. N Eng/ J Med 2012;366:1227-39.

3 Stitt AW, Gardiner TA, Archer DB. Histological and ultrastructural investigation of retinal microaneurysm development in diabetic patients. Br J Ophthalmol 1995;79:362-7.

4 Moore J, Bagley S, Ireland $\mathrm{G}$, et al. Three dimensional analysis of microaneurysms in the human diabetic retina. J Anat 1999:194:89-100.

5 Spaide RF, Fujimoto JG, Waheed NK, et al. Optical coherence tomography angiography. Prog Retin Eye Res 2018;64:1-55.

6 Gardner TW, Antonetti DA. Novel potential mechanisms for diabetic macular edema: leveraging new investigational approaches. Curr Diab Rep 2008;8:263-9.

7 Klein R, Klein BE, Moss SE, et al. The wisconsin epidemiologic study of diabetic retinopathy. III. Prevalence and risk of diabetic retinopathy when age at diagnosis is 30 or more years. Arch Ophthalmol 1984;102:527-32.

8 Ribeiro ML, Nunes SG, Cunha-Vaz JG. Microaneurysm turnover at the macula predicts risk of development of clinically significant macular edema in persons with mild nonproliferative diabetic retinopathy. Diabetes Care 2013;36:1254-9.

9 Flaxel CJ, Adelman RA, Bailey ST, et al. Diabetic Retinopathy Preferred Practice Pattern®. Ophthalmology 2020;127:66-145.

10 Klein R, Meuer SM, Moss SE, et al. Retinal microaneurysm counts and 10-year progression of diabetic retinopathy. Arch Ophthalmol 1995;113:1386-91.

11 Hellstedt T, Vesti E, Immonen I. Identification of individual microaneurysms: a comparison between fluorescein angiograms and red-free and colour photographs. Graefes Arch Clin Exp Ophthalmol 1996;234:S13-17.

12 Spaide RF, Klancnik JMJ, Cooney MJ. Retinal vascular layers imaged by fluorescein angiography and optical coherence tomography angiography. JAMA Ophthalmol 2015; 133:45-50.

13 Inoue $\mathrm{M}$, Jung JJ, Balaratnasingam C, et al. A comparison between optical coherence tomography angiography and fluorescein angiography for the imaging of type 1 neovascularization. Invest Ophthalmol Vis Sci 2016;57:314-23.

14 Liu G, Xu D, Wang F. New insights into diabetic retinopathy by OCT angiography. Diabetes Res Clin Pract 2018;142:243-53.

15 Corvi F, Pellegrini M, Erba S, et al. Reproducibility of vessel density, fractal dimension, and foveal avascular zone using 7 different optical coherence tomography angiography devices. Am J Ophthalmol 2018;186:25-31.

16 Corvi F, Cozzi M, Barbolini E, et al. Comparison between several optical coherence tomography angiography devices and indocyanine green angiography of choroidal neovascularization. Retina 2019; Epub ahead of print.

17 Matsunaga D, Yi J, Puliafito CA, et al. OCT angiography in healthy human subjects. Ophthalmic Surg Lasers Imaging Retina 2014;45:510-5.

18 Schreur V, Domanian A, Liefers B, et al. Morphological and topographical appearance of microaneurysms on optical coherence tomography angiography. $\mathrm{Br} J \mathrm{Ophthalmol}$ 2018; Epub ahead of print.

19 Couturier A, Mane V, Bonnin S, et al. Capillary plexus anomalies in diabetic retinopathy on optical coherence tomography angiography. Retina 2015;35:2384-91.

20 Nakao S, Yoshida S, Kaizu Y, et al. Microaneurysm detection in diabetic retinopathy using OCT angiography may depend on intramicroaneurysmal turbulence. Ophthalmol Retina 2018;2:1171-3.

21 Hasegawa N, Nozaki M, Takase N, et al. New insights into microaneurysms in the deep capillary plexus detected by optical coherence tomography angiography in diabetic macular edema. Invest Ophthalmol Vis Sci 2016;57:348-55.

22 Kaizu Y, Nakao S, Wada I, et al. Microaneurysm imaging using multiple en face OCT angiography image averaging: morphology and visualization. Ophthalmol Retina 2020:4:175-86. 